\title{
Conjugation and deconjugation of ubiquitin regulating the destiny of proteins
}

\author{
Kwang-Hyun Baek \\ Department of Microbiology \\ College of Medicine, Pochon CHA University \\ Cell and Gene Therapy Research Institute \\ CHA General Hospital, Seoul 135-081, Korea \\ Tel, 82-2-3468-3197; Fax, 82-2-3468-3264; \\ E-mail, baek@cha.ac.kr
}

Accepted 22 January 2003

Abbreviations: DUB, deubiquitinating enzyme; Ub, ubiquitin

\section{Overview}

The homeostasis for a number of cellular proteins is regulated by not only phosphorylation and dephosphorylation, but also ubiquitination and deubiquitination. A number of proteins involved in the degradation of polypeptides have been isolated in various eukaryotic organisms from Saccharomyces cerevisiae to human. Recently, several deubiquitinating enzymes, classified into either the $\mathrm{Ub}$ C-terminal hydrolase (UCH) or the Ub-specific processing protease (UBP), have been reported. It has been shown that they contain conserved domains including Cys, His, and Asp residues throughout the enzyme. These proteins have been demonstrated that Cys and His domains are critical for deubiquitinating enzymatic activity. Recently, we have shown that the Asp domain localized between Cys and His domains is also essential for cleaving the ubiquitin from protein substrates. Mouse deubiquitinating enzymes including DUB-1, DUB-2, and DUB-2A have been isolated and they showed the expression specificity. Of these, DUB1 and DUB-2 are expressed in lymphocytes depending on the presence of cytokines (interleukin-3 in B-lymphocytes and interleukin-2 in Tlymphocytes, respectively), indicating that they are involved in cytokine signaling pathways. Isolation of all putative DUBs will help to identify their substrates and to regulate the homeostasis of cellular proteins, especially in proliferative cells.

Keywords: lymphocytes; protein processing, posttranslational; ubiquitin
Ubiquitin-mediated protein degradation pathway

The concepts of Yin and Yang have provided the intellectual framework of people in Asian countries including Korea, China, and Japan, especially in the fields of biology and medicine. By philosophical analogy, a number of cellular proteins are regulated by not only phosphorylation by kinases, but also dephosphorylation by phosphatases. Recently, a similar counterbalancing action for a number of proteins in eukaryotic organisms is established, ubiquitination by ubiquitin conjugating enzymes and deubiquitination by deubiquitinating enzymes (Figure 1). This includes most of oncoproteins and signaling components involved in receptor tyrosine kinase-mediated signal transduction pathways. Ubiquitin (Ub), a small polypeptide of 76 amino acids, is conjugated to its specific target protein by hierarchical enzymatic reactions (Figure 2). The ubiquitin-activating enzymes (E1) in this cascade reaction activate the ubiquitin molecule in an ATP-dependent manner. This ubiquitin is then transferred to ubiquitin-conjugating enzymes (E2). These enzymes function with ubiquitin-protein ligases (E3) to mediate the final transfer of the ubiquitin to the lysine residues in its protein targets (Hochstrasser, 1996; Wilkinson, 1997; Ciechanover et al., 2000; Weissman, 2001). In yeast, there has been a report that a novel ubiquitination factor (E4) is essential for efficient multiubiquitination (Koegl et al., 1999). Ubi-

Phosphorylation (kinases)

Ubiquitination (ubiquitin conjugating enzymes)

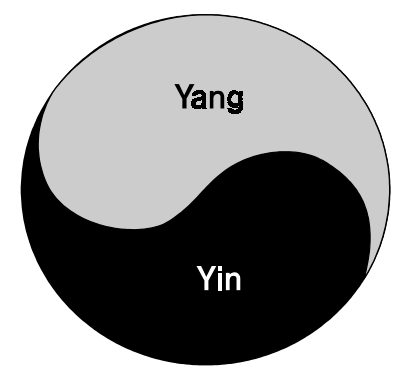

Dephosphorylation (phosphatases)

Deubiquitination (deubiquitinating enzymes)

Figure 1. Like the Yin and Yang mythology in Asian countries, cellular proteins are regulated by not only phosphorylation by kinases and dephosphorylation by phosphatases, but also ubiquitination by ubiquitin conjugating enzymes and deubiquitination by deubiquitinating enzymes. 
quitinated proteins are recognized and degraded by the $26 \mathrm{~S}$ proteasome, which is composed of a proteolytic core catalytic complex (20S) and two 19S regulatory complexes leading to an enormous size (2.5 MDa). These complexes associate together in an ATP-dependent manner (Etlinger and Goldberg, 1977; Hershko, 1996; Holzl et al., 2000; Leggett et al., 2002).

Several classes of proteasome inhibitors have been identified including peptide aldehydes (MG132, PSI, and CEP1612), peptide vinyl sulfone (NLVS), peptide epoxyketones (Epoxomicin and Dihydroeponemycin), peptide boronates (PS-341 and DFLB), and lactacystin and derivatives (lactacystin, omuralide, and PS519) (Rock et al., 1994; Dick et al., 1996; Tsubuki et al., 1996; Adams et al., 1998; Corey and Li, 1999; Kisselev and Goldberg, 2001). These inhibitors have been used for identifying a number of substrates of the ubiquitin-proteasome pathway and are important for investigating the pathway due to the significance of proteasome in many aspects of cellular protein targets in eukaryotic organisms.

\section{Ubiquitin cleavage by deubiquitinating enzymes}

The conjugation of ubiquitin molecule to its specific target proteins is a reversible process. Deubiquitinating enzymes play a role in cleaving the ubiquitin from protein substrates (Figure 2). This cleavage is involved in a number of biologically important processes including preimplantation, growth and differentiation, oncogenesis, cell cycle progression, transcriptional ac-

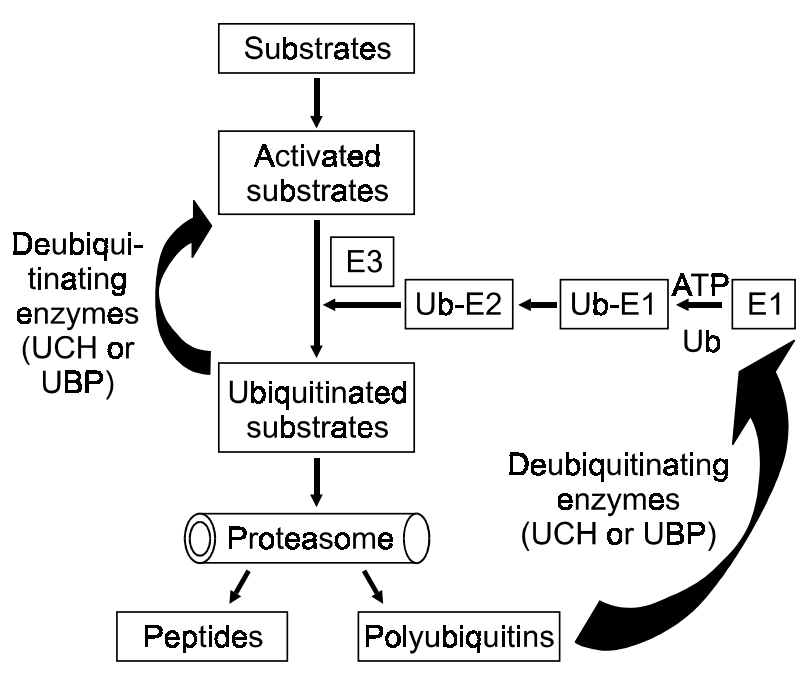

Figure 2. The ubiquitin-proteasome system. Ubiquitin-mediated protein degradation is regulated by a series of Ub-conjugating enzymes (ubiquitin activating enzyme E1, ubiquitin conjugating enzyme E2, and ubiquitin ligase E3) and deubiquitinating enzymes (UCH or UBP). Ubiquitinated protein substrates are degraded by the $26 \mathrm{~S}$ proteasome. tivation, and signal transduction (Papa and Hochstrasser, 1993; Moazed and Johnson, 1996; Zhu et al., 1996; Cadavid et al., 2000; Pantaleon et al., 2001; Park et al., 2002). A number of genes encoding a deubiquitinating enzyme have been identified in various eukaryotic organisms and are classified into two groups, the Ub-specific processing protease (UBP) family and the Ub C-terminal hydrolase $(\mathrm{UCH})$ family (Wilkinson, 1997). In general, UBP family members, regulating signal transduction, growth, and development, are responsible for cleaving the ubiquitin from larger proteins and disassembling the polyubiquitin chains. UCH enzymes, playing important roles in development and neural function, are papain-like proteases and are responsible for removing the ubiquitin from small peptides or larger substrates with a flexible peptide linking the C-terminal domain (Wilkinson, 2000).

Seventeen putative deubiquitinating enzymes have been isolated in the genome of Saccharomyces cerevisiae (Amerik et al., 2000). This leads to the possibility that a number of deubiquitinating enzymes may exist in higher eukaryotic organisms including human. Recent studies revealed that several deubiquitinating enzymes are involved in cell proliferation and development. This includes the murine unp gene that is tumorigenic in nude mice (Gray et al., 1995), the Drosophila faf gene that determines cell growth and differentiation during eye development in fruit flies (Huang et al., 1995), and the mammalian tre-2 oncoprotein that shows transforming activity in NIH3T3 fibroblast (Papa and Hochstrasser, 1993). However, the precise molecular mechanisms for these enzymes are not well-studied due to the technical difficulty in purifying ubiquitinated substrates for them. In order to develop pharmacological agents to activate or inhibit proteasomal degradation, isolation and characterization of substrates for deubiquitinating enzymes are required. The human and mouse genome sequencing projects will help to identify genes encoding a putative deubiquitinating enzyme and their substrates in both organisms and are expected to be the largest family members in the ubiquitin system.

\section{Disorders caused by abnormal ubiquitin- mediated protein degradation pathway}

It has been demonstrated that aberrant ubiquitinproteasome pathway can lead to a variety of disorders including neurodegenerative diseases such as Parkinson's disease and Alzheimer's disease (Layfield et al., 2001). In general, protein aggregation is a common symptom for most of the chronic neurodegenerative diseases in humans. The intraneuronal inclusions in many of these diseases include excessive ubiquitin-conjugated proteins, suggesting aberrant ubiquitin-mediated protein degradation. 
Parkinson's disease is one of common neurodegenerative disorders characterized by the loss of dopaminergic neurons in the midbrain and the presence of Lewy bodies, cytoplasmic inclusions that are abundantly enriched in ubiquitin (Chung et al., 2001). It has been reported that mutations in the parkin gene, encoding an E3 ubiquitin-protein ligase, can cause autosomal-recessive form of juvenile Parkinson's disease (Kitada et al., 1998). Recent studies revealed that parkin protein interacts and ubiquitinates the $\alpha$-synuclein-interacting protein, synphilin-1 (Chung et al., 2001). Therefore, the aberrant function of parkin, $\alpha$-synuclein, or synphilin-1 can lead to protein aggregation in Lewy bodies. Another example of protein aggregation is shown in Alzheimer's disease. Mutations in presenillins may lead to early onset forms of Alzheimer's disease due to the disruption of the maturation of the $\beta$-amyloid precursor protein (Checler et al., 2000).

Fanconi anemia (FA) is an autosomal recessive cancer susceptibility disease characterized by multiple congenital anomalies, bone marrow failure, and mitomycin sensitivity (Taniguchi et al., 2002; Gregory et al., 2003). A novel FA pathway, required for the normal cellular response to DNA damage, has been suggested (Wilson et al., 2001). Several FA genes including FANCA, FANCC, FANCD2, FANCE, FANCF, and FANCG have been isolated and their gene products interact in a cellular FA pathway (Gregory et al., 2003). It has been demonstrated that FANCA, FANCC, FANCE, FANCF, and FANCG proteins assemble in a multisubunit complex (Medhurst et al., 2001), and this complex is required for the monoubiquitination of the FANCD2 protein (Wilson et al., 2001). This suggests that the complex plays a role as an ubiquitin ligase. Therefore, the aberrant function of any FA protein in a multisubunit complex may lead to severe clinical damages such as anemia and bone marrow failure.

\section{Deubiquitinating enzymes involved in cytokine signaling pathways}

In response to cytokine stimulation, cells change their cellular states. Cytokines induce the expression of specific genes that encode regulatory proteins regulating protein degradation (Akiyama et al., 1994). Recently, the subfamily members of deubiquitinating enzymes (DUB-1, DUB-2, and DUB-2A) have been isolated and have been shown that they are induced by different cytokines in murine hematopoietic cells (Zhu et al., 1996; Zhu et al., 1997; Baek et al., 2001). $D \cup B-1$, induced by interleukin-3, interleukin-5, or granulocyte-macrophage colony stimulating factor, is an immediate-early gene, and is expressed in murine hematopoietic progenitor cell line $\mathrm{Ba} / \mathrm{F3}$ (Figure 3; Zhu et al., 1996). DUB-2, is also an immediate-early

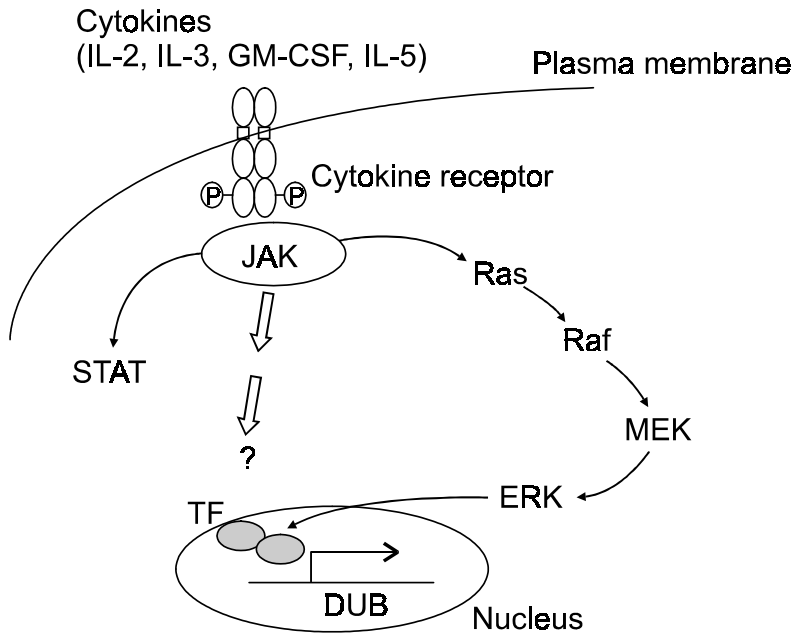

Figure 3. Cytokine-induced JAK activation. JAK kinase is activated upon binding of cytokines to the receptors. They then phosphorylate the receptors on tyrosines, which in turn allow the activation of STAT and Ras signaling. JAK-dependent, STAT-independent pathway is required for the induction of genes encoding murine hematopoietic deubiquitinating enzymes (DUB-1 and DUB-2).

gene, which is induced by interleukin-2 in murine hematopoietic progenitor cell line CTLL (Figure 3; Zhu et al., 1997).

It has been demonstrated that JAK2 kinase and Ras-Raf-MEK-ERK kinase signaling pathway are essential for induction of the murine DUB-1 gene, even though the presence of another signaling pathway may be involved (Figure 3; Zhu et al., 1997). Interestingly, the minimal interleukin-3 response element of DUB-1 gene contains cytokine-inducible enhancer activity, but lacks a consensus sequence for STAT binding. This indicates that DUB-1 is expressed in a JAK2-dependent pathway but STAT5-independent pathway (Zhu et al., 1997). Since a number of receptor tyrosine kinases are regulated not only by phosphorylation and dephosphorylation, but also by ubiquitination and deubiquitination, it is plausible that signaling pathways mediated by these receptors can be modulated by the ubiquitination status of them. In addition, the amount of signaling components, which are to be conjugated with the ubiquitin for proteasomal degradation, can also modulate signaling pathways. Therefore, finding the molecular mechanisms for the regulation of protein degradation via ubiquitination and deubiquitination in RTK-mediated signal transduction pathways will contribute to understanding the regulation of cell proliferation and differentiation.

\section{Structural conservation of deubiquitinating enzymes}

Like ubiquitin conjugating enzymes, which are con- 


\begin{tabular}{|c|c|c|c|c|c|}
\hline 215 & GLKNQGATCYMNSLLQTLF & $\begin{array}{ll}F \quad 234 \\
\end{array}$ & HAUSP & (human) & \multirow{5}{*}{ Cys domain } \\
\hline 81 & GLQNMGNTCYVNASLQCLTY & Y 100 & USP17 & (human) & \\
\hline 52 & GLQNTGNSCYLNAALQCLTH & 71 & DUB-1 & (mouse) & \\
\hline 52 & GLQNTGNSCYLNAALQCLTF & 71 & DUB-2 & (mouse) & \\
\hline \multirow[t]{2}{*}{52} & GLQNTGNSCYLNAALQCLTH & 71 & DUB-2A & (mouse) & \\
\hline & * & & & & \multirow{6}{*}{ His domain } \\
\hline 451 & HAVLVHSGDNHG-GHYV & 466 & HAUSP & (human) & \\
\hline 320 & YAVLVHAGWSCHNGHYF & 336 & USP17 & (human) & \\
\hline 293 & YAVLVHDGATSHSGHYF & 309 & DUB-1 & (mouse) & \\
\hline 293 & YAVLVHEGATCHSGHYF & 309 & DUB-2 & (mouse) & \\
\hline \multirow[t]{2}{*}{293} & YAVLVHEGATCHSGHYF & 309 & DUB-2A & (mouse) & \\
\hline & * & & & & \multirow{6}{*}{ Asp domain } \\
\hline 475 & GKWCKFDDDVVSRCTKEE & 492 & HAUSP & (human) & \\
\hline 344 & GQWYKMDDAEVTASSITS & 361 & USP17 & (human) & \\
\hline 317 & GAWYKMDDTKVTSCDVTS & 334 & DUB-1 & (mouse) & \\
\hline 317 & GAWYKMDDTKVTSCDVTS & 334 & DUB-2 & (mouse) & \\
\hline 317 & GAWYKMDDTKVTSCDVTS & 334 & DUB-2A & (mouse) & \\
\hline
\end{tabular}

Figure 4. Alignment of conserved amino acid sequences for deubiquitinating enzymes derived from GenBank accession numbers, HAUSP (Z72499), USP17 (D38378), DUB-1 (Q61068), DUB-2 (AAB95194), and DUB-2A (AAK84135) using MegAlign software (Clustal method) from DNASTAR (LASERGENE). Conserved amino acid residues for putative catalytic triad according to the structural study are indicated with asterisks.

served from yeast to human, the UCHs of deubiquitinating enzymes are also highly conserved (Johnston et al., 1999). The UBPs contain divergent sequences throughout the enzymes, even though the alignment of them indicates that the six DUB homology domains are found (D'Andrea and Pellman, 1998; Lockington and Kelly, 2001). Of these, three conserved domains (cysteine, histidine, and aspartic acid residues) comprise the catalytic triad for an active site of the enzyme (Figure 4; Baek et al., 2001; $\mathrm{Hu}$ et al., 2002). It has been suggested that the divergence of amino acid sequences for UBPs is involved in a broad range of substrate specificity (Papa and Hochstrasser, 1993; Wilkinson, 1997). Site-directed mutagenesis experiments for these conserved amino acids revealed that they are required for deubiquitinating enzyme activity (Figure 5B; Papa and Hochstrasser, 1993; Huang et al., 1995; Baek et al., 2001; Lee et al., 2001; Hu et al., 2002). The deletion analysis of the carboxy-terminal region for deubiquitinating enzyme subfamily members (DUB-1, DUB-2 and DUB-2A) showed no effect for the deubiquitinating enzyme activity (Baek et al. 2001; unpublished data). This suggests that the carboxy-terminal region of these enzymes may be involved in substrate specificity, protein-protein interaction, and localization. Recently, the crystal structure for the catalytic domain of HAUSP, one of UBPs that deubiquitinates and stabilizes the tumor suppressor p53, has been studied (Hu et al., 2002). This study showed that the catalytic core domain of HAUSP enzyme binds ubiquitin aldehyde, leading to a dramatic conformational change

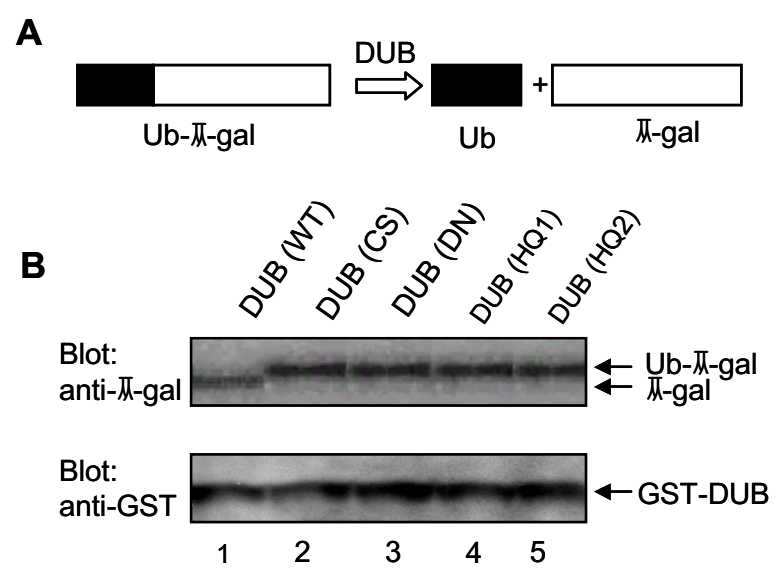

Figure 5. A. Ub- $\beta$-gal assay for deubiquitination of ubiquitin- $\beta$-galactosidase (Ub-Met- $\beta$-gal) fusion protein expressed in Escherichia coli. $B$. The upper panel is an immunoblot using anti- $\beta$-gal antiserum. The co-expressed plasmids are pGEX-DUB wild-type (lane 1), pGEX-DUB CS (lane 2), pGEX-DUB DN (lane 3), pGEX-DUB HQ1 (lane 4), and pGEX-DUB HQ2 (lane 5). The lower panel is an immunoblot using an anti-GST monoclonal antibody.

in the active site similar to one for HAUSP binding to its substrate (Hu et al., 2002). The detailed molecular mechanisms for their binding should be investigated in order to create pharmaceutical reagents that target to these mechanisms in signal transduction pathways involved in cell proliferation and differentiation. 


\section{Isolation of putative deubiquitinating enzymes and future directions}

More than 90 putative deubiquitinating enzymes have been identified by the human genome sequencing project, making them one of the largest family of enzymes in the ubiquitination and deubiquitination system (Chung and Baek, 1999). As mentioned above, the UBP enzymes contain high homology domains that surround the catalytic Cys, His and Asp residues. Using bioinformatics, we previously identified a deubiquitinating enzyme gene, $U B H 1$, in mouse testis (Baek et al., 2002). In order to screen putative deubiquitinating enzymes containing a conserved aspartic acid motif for the formation of the oxyanion hole in mouse ( $\mathrm{Hu}$ et al., 2002), databases from GenBank were accessed and searched using the BLAST algorithm (Altschul et al., 1997) at the National Center for Biotechnology Information (NCBI, http://www.ncbi. nlm.nih.gov/BLAST/). We previously demonstrated for the first time that the conserved aspartic acid residue in the oxyanion hole of mouse DUB-1, DUB-2 or DUB-2A, which is expressed specifically in either $B$ lymphocytes or $T$ lymphocytes, is required for the enzymatic activity (Baek et al., 2001; Lee et al., 2001). By screening the databases, we found a total number of 11 genes encoding a deubiquitinating enzyme including DUB-1, DUB-2, and DUB-2A (Baek et al., 2002). One of them was cloned and named as UBH1 (Baek et al., 2002). The sequence analysis for these genes suggests a similar mechanism for all UBP enzymes in the formation of the oxyanion hole and catalysis (Hu et al., 2002). The detailed mechanism should be elucidated in order to speculate pharmacological manipulation for UBP-related diseases. Several other putative genes encoding a deubiquitinating enzyme have been identified by this method and they are under investigation.

A novel ubiquitin-specific protease gene UBP41 was isolated from the chick skeletal muscle cDNA library and the deubiquitinating enzyme activity was analyzed by Ub- $\beta$-gal assay in Escherichia coli (Figure $5 \mathrm{~A}$; Baek et al., 1997). Recently, 23 deubiquitinating enzymes including the tumor suppressor CYLD1 have been identified by chemistry-based functional proteomics in EL4 cells (Borodovsky et al., 2002). In addition, with information obtained from genome sequencing projects in various living organisms including human and mouse, a number of genes encoding a deubiquitinating enzyme will be identified by various methods. While the physiological functions for most of deubiquitinating enzymes in vivo remain unknown, several features of them suggest that they play an important role in cell proliferation and differentiation. Therefore, finding specific substrates and the molecular mechanism of deubiquitination by these enzymes will elucidate their physiological roles. Ob- viously, this will give some insights on how these enzymes may function for intracellular processes including signal transduction pathways, cell cycle progression, transcriptional activation, antigen presentation, apoptosis (or programmed cell death), and DNA repair within the cell.

\section{Acknowledgement}

I wish to thank the members of Baek's laboratory and Cell and Gene Therapy Research Institute at CHA General Hospital, Pochon CHA University. This work was supported by the Korea Institute of S\&T Evaluation and Planning (M102KL010001-02K120102230).

\section{References}

Adams J, Behnke $M$, Chen S, Cruickshank AA, Dick LR, Grenier L, Klunder JM, Ma YT, Plamondon L, Stein RL. Potent and selective inhibitors of the proteasome: dipeptidyl boronic acids. Bioorg Med Chem Lett 1998;8:333-8

Akiyama K, Yokota K, Kawaka S, Shimbara N, Tamura T, Akioka H, Nothwang HG, Noda C, Tanaka K, Ichihara A. cDNA cloning and interferon gamma down-regulator of proteasomal subunits $X$ and $Y$. Science 1994;26:1231-4

Altschul SF, Madden TL, Schaffer AA, Zhang J, Zhang Z, Miller W, Lipman DJ. Gapped BLAST and PSI-BLAST: a new generation of protein database search programs. Nucleic Acids Res 1997;25:3389-402

Amerik AY, Li SJ, Hochstrasser M. Analysis of the deubiquitinating enzymes of the yeast Saccharomyces cerevisiae. J Biol Chem 2000;381:981-92

Baek KH, Mondoux MA, Jaster R, Fire-Levin E, D'Andrea, AD. DUB-2A, a new member of the DUB subfamily of hematopoietic deubiquitinating enzymes. Blood 2001;98:63642

Baek KH, Park KH, Kim YS, Kim MS, Choi HK. Molecular cloning and complete cDNA sequence of UBH1 in mouse testis. DNA Seq 2002;13:145-8

Baek SH, Choi KS, Yoo YJ, Cho JM, Baker RT, Tanaka $\mathrm{K}$, Chung $\mathrm{CH}$. Molecular cloning of a novel ubiquitin-specific protease, UBP41, with isopeptidase activity in chick skeletal muscle. J Biol Chem 1997;272:25560-5

Borodovsky A, Ovaa H, Kolli N, Gan-Erdene T, Wilkinson $\mathrm{KD}$, Ploegh HL, Kessler BM. Chemistry-based functional proteomics reveals novel members of the deubiquitinating enzyme family. Chem Biol 2002;9:1149-59

Cadavid ALM, Ginzel A, Fischer JA. The function of the Drosophila Fat facets deubiquitinating enzyme in limiting photoreceptor cell number is intimately associated with endocytosis. Development 2000;127:1727-36

Checler F, da Costa CA, Ancolio K, Chevallier N, LopezPerez E, Marambaud P. Role of the proteasome in Alzheimer's disease. Biochim Biophys Acta 2000;1502:133-8

Chung $\mathrm{CH}$, Baek SH. Deubiquitinating enzymes: their diver- 
sity and emerging roles. Biochem Biophys Res Commun 1999;266:633-40

Chung KK, Zhang $Y$, Lim KL, Tanaka $Y$, Huang $H$, Gao J, Ross CA, Dawson VL, Dawson TM. Parkin ubiquitinates the alpha-synuclein-interacting protein, synphilin-1: implications for Lewy-body formation in Parkinson disease. Nat Med 2001;7:1144-50

Ciechanover A. The ubiquitin-proteasome pathway: on protein death and cell life. EMBO J 1998;17:7151-60

Ciechanover A, Orian A, Schwartz AL. Ubiquitin-mediated proteolysis: biological regulation via destruction. Bioessays 2000;22:442-51

Corey EJ, Li WD. Total synthesis and biological activity of lactacystin, omuralide and analogs. Chem Pharm Bull 1999; 47:1-10

D'Andrea $A D$, Pellman D. Deubiquitinating enzymes: a new class of biological regulators. Crit Rev Biochem Mol Biol 1998;33:337-52

Dick LR, Cruikshank AA, Grenier L, Melandri FD, Nunes SL, Stein RL. Mechanistic studies on the inactivation of the proteasome by lactacystin a central role for clasto-lactacystin $\beta$-lactone. J Biol Chem 1996;271:7273-6

Etlinger JD, Goldberg AL. A soluble ATP-dependent proteolytic system responsible for the degradation of abnormal proteins in reticulocytes. Proc Natl Acad Sci USA 1977;74: 54-8

Gray DA, Inazawa J, Gupta K, Wong A, Ueda R, Tahahashi T. Elevated expression of Unph, a proto-oncogene at 3p21.3, in human lung tumors. Oncogene 1995;10:2179-83

Gregory RC, Taniguchi T, D'Andrea AD. Regulation of the Fanconi anemia pathway by monoubiquitination. Semin Cancer Biol 2003:13:77-82

Hershko A. Lessons from the discovery of the ubiquitin system. Trends Biochem Sci 1996;21:445-9

Hochstrasser M. Protein degradation or regulation: Ub the judge. Cell 1996;84:813-5

Holzl H, Kapelari B, Kellermann J, Seemuller E, Sumegi M, Udvardy A, Medalia O, Sperling J, Muller SA, Engel A, Baumeister W. The regulatory complex of Drosophila melanogaster $26 \mathrm{~S}$ proteasomes. Subunit composition and localization of a deubiquitylating enzyme. J Cell Biol 2000; 150:119-30

Hu M, Li P, Li M, Li W, Yao T, Wu JW, Gu W, Cohen RE, Shi Y. Crystal structure of a UBP-family deubiquitinating enzyme in isolation and in complex with ubiquitin aldehyde. Cell 2002;111:1041-54

Huang Y, Baker RT, Fischer-Vize JA. Control of cell fate by a deubiquitinating enzyme encoded by the fat facets gene. Science 1995;270:1828-31

Johnston SC, Riddle SM, Cohen RE, Hill CP. Structural basis for the specificity of ubiquitin C-terminal hydrolases. EMBO J 1999;18:3877-87

Kisselev AF, Goldberg AL. Proteasome inhibitors: from research tools to drug candidates. Chem Biol 2001;8:739-58

Kitada S, Kojima K, Shimokata K, Ogishima T, Ito A. Glu- tamate residues required for substrate binding and cleavage activity in mitochondrial processing peptidase. J Biol Chem 1998;273:32547-53

Koegl M, Hoppe T, Schlenker S, Ulrich HD, Mayer TU, Jentsch S. A novel ubiquitination factor, E4, is involved in multiubiquitin chain assembly. Cell 1999;96:635-44

Layfield R, Alban A, Mayer RJ, Lowe J. The ubiquitin protein catabolic disorders. Neuropathol Appl Neurobiol 2001;27:1719

Lee JH, Kim YS, Kim M, Baek KH. Critical regions for deubiquitinating activity of DUB-2 expressed in T-lymphocytes. Am J Hematol 2001;67:270-2

Leggett DS, Hanna J, Borodovsky An, Crosas B, Schmidt M, Baker RT, Walz T, Ploegh H, Finley D. Multiple associated proteins regulate proteasome structure and function. Mol Cell 2002;10:495-507

Lockington RA, Kelly JM. Carbon catabolite repression in Aspergillus nidulans involves deubiquitination. Mol Microbiol 2001;40:1311-21

Medhurst AL, Huber PA, Waisfisz Q, de Winter JP, Mathew CG. Direct interactions of the five known Fanconi anaemia proteins suggest a common functional pathway. Hum Mol Genet 2001;10:423-9

Moazed $D$, Johnson AD. A deubiquitinating enzyme interacts with SIR4 and regulates silencing in S. cerevisiae. Cell 1996; 86:667-77

Pantaleon M, Kanai-Azuma M, Mattick JS, Kaibuchi K, Kaye $\mathrm{PL}$, Wood SA. FAM deubiquitylating enzyme is essential for preimplantation mouse embryo development. Mech Dev 2001; 109:151-60

Papa FR, Hochstrasser M. The yeast DOA4 gene encodes a deubiquitinating enzyme related to a product of the human tre-2 oncogene. Nature 1993;366:313-9

Park KC, Kim JH, Choi EJ, Min SW, Rhee S, Baek SH, Chung SS, Bang O, Park D, Chiba T, Tanaka K, Chung $\mathrm{CH}$. Antagonistic regulation of myogenesis by two deubiquitinating enzymes, UBP45 and UBP69. Proc Natl Acad Sci USA 2002;99:9733-8

Rock KL, Gramm C, Rothstein L, Clark K, Stein R, Dick L, Hwang D, Goldberg AL. Inhibitiors of the proteasome block the degeneration of most cell proteins and the generation of peptides presented on MHC class I molecules. Cell 1994; 78:761-71

Taniguchi $T$, D'Andrea $A D$. The Fanconi anemia protein, FANCE, promotes the nuclear accumulation of FANCC. Blood 2002;100:2457-62

Tsubuki S, Saito Y, Tomioka M, Ito H, Kawashima S. Differential inhibition of calpain and proteasome activities by peptidyl aldehydes of di-leucine and tri-leucine. J Biochem 1996:119:572-6

Weissman AM. Themes and variations on ubiquitylation. Nat Rev Mol Cell Biol 2001;2:169-78

Wilkinson KD. Regulation of ubiquitin-dependent processes by deubiquitinating enzymes. FASEB J 1997;11:1245-56

Wilkinson KD. Ubiquitination and deubiquitination: targeting of proteins for degradation by the proteasome. Semin Cell 
Dev Biol 2000;11:141-8

Wilson JB, Johnson MA, Stuckert AP, Trueman KL, May $S$, Bryant PE, Meyn RE, D'Andrea AD, Jones NJ. The Chinese hamster FANCG/XRCC9 mutant NM3 fails to express the monoubiquitinated form of the FANCD2 protein, is hypersensitive to a range of DNA damaging agents and exhibits a normal level of spontaneous sister chromatid exchange. Carcinogenesis 2001;22:1939-46
Zhu Y, Carroll M, Papa FR, Hochstrasser M, D'Andrea AD. DUB-1, a deubiquitinating enzyme with growth suppressing activity. Proc Natl Acad Sci USA 1996;93:3275-9

Zhu Y, Lambert K, Corless C, Copeland NG, Gilbert DJ, Jenkins NA, D'Andrea AD. DUB-2 is a member of a novel family of cytokine-inducible deubiquitinating enzymes. J Biol Chem 1997;272:51-7 